\title{
AVR SYSTEM ANALYSIS AND SIMULATION BY USING FOPID AND PARAMETERS VARIATION EFFECTS
}

\author{
Choppadandi Srikanth ${ }^{l}$, Dola Gobinda Padhan $^{l}$, N. Kiran Kumar ${ }^{2}$. \\ ${ }^{1}$ Department of Electrical and Electronics Engineering, GRIET, Hyderabad. \\ ${ }^{2}$ Department of Electrical and Electronice Engineering, Vardhaman College of Engineering, Hyderabad
}

\begin{abstract}
The FOPID control units for an AVR system with a fractional filter are a unique fractional order. The main responsibility for controlling the reactive power and voltage level is an automated tensile regulator (AVR). PID controller, sensor, exciter, and stabiliser or amplifier are used for the module system. The system is designed according to state-space technology. The proposed controller must have seven independent parameters. A comparison of the published study with optimal adjusted AVR PID and FOPID controls also shows that the proposed controller is superior. The recommended controls derived from the bode analysis with their frequency response characteristics are shown. Finally, the resilience of the controller design is individually examined for both the parameter uncertainties of AVR system and outside storages introduced into AVR system. Given the overall results presented there are clear improvements to the performance of AVR system by a fractional filter in a proposed FOPID controller, and that the AVR system may successfully be applied to the suggested control.
\end{abstract}

\section{Introduction}

Reactive power is the foremost primary aspects for use and design in power systems. A constant tension output is the reactive force balance in the system. By shunting the reactive power injection, by transferring reactive power into the system via tap changes and lowering induction lane reagents through a succession of condensers, one may define the most common reactive power and voltage management systems. AVR systems are commonly used in exciter control systems. Major technique of the reactive power control is AVR generator excitation control. In typical operational conditions, AVR shall maintain the generator terminal voltage constant at varied load levels[1]. The Terminal Voltage Error is used to modify the voltage in AVR loop for an axle control system to administer the terminal-voltage[2]. Four important components - amplifier, sensor, exciter, and generator - are included in a controller exciter. Several articles discussed the AVR system control principles[2-5]. A Real energy losses in electricity generators are a general difficulty to solve. Researchers are studying automated voltage control units (AVR) to reduce these power losses in the power system. The major goal of this kind of AVR systems is to keep generator voltage at correct level highly precise. This precision enhances the life cycle of power system rated voltage equipment. The AVR system's stability and strength have a considerable impact on the power system's security[1]. Therefore, an efficiency of AVR system continues to be an important topic for the development of new control systems. In particular, many studies have been done to improve the AVR system's transitional reaction to the stability limitations. Researchers have devised several control ways to control the AVR generator voltage so far in an effort to get an enhanced system response. Some of the control techniques presented are the PID fractional order control (PRD) [2-4] [5,6]. Despite these enormous modifications, the scholars focus more on PID controller or its amalgamations because to its straightforward control structure and effectiveness.

PID controller parameters, like other controllers, must be adequately tailored to deliver a good system response. In addition, PID controller settings must be optimally modified in order to reach the best system answer. However, depending on industrial use, this perfectly adjusting method presents a serious problem. 
Meta-heuristic optimization algorithms have, in recent years, been favoured in conventional tuning procedures like Ziegler-Nichols and Cohen-Coon, as in most circumstances the results are not optimal[3,10]. Researchers have built AVR System PID controllers that use developmental algorithms like other industrial applications. The FOPID controller is also used in addition to the PID controller to improve generator voltage quality with versatile design options in many technological fields. With two additional parameters than the traditional PID controller, FOPID provides greater design freedom, but five different parameters are more difficult to design for the controller. With regard to the transient responsiveness characters such as over-percentage shots, set-off times, peak times and rising times and steady-state errors, the scientists have aimed to increase their AVR system response. The researchers' goal is in the same direction however, as neither approaches can ensure that the best controller parameter for the AVR system is found, they prefer alternative kinds of algorithms. In other words, using a new algorithm can improve the settings of the controller to improve the system response to AVR. Also, in their above-described optimization techniques researchers have examined many types of goal functions to enhance AVR system response. AVR's [2-5,9,1013,13,18,16-18,20-23] system also includes user-defined target functions, which combine the surplus percentage, setup time, peak time, time increases, stable state error and gain margin. [2] offers an over-hitch percentage, settlement time, time increment, and continuous state mistakes as a typical user-defined target function. This objective function was frequently employed by the scholars to provide fair comparison in between their method and accessible literature.

\section{AVR System Model}

AVR system, comprising amplifications, exciters, generator and sensor components, [1,2,28], for the output voltage from the synchronised generator stationary to accurate levels. As in Fig.1, AVR system without controller can be built with each component characterised by transfer of first-order function with ' $\mathrm{K}$ ' gain and the time constant' $\mathrm{t}$ in the AVR system. The $\mathrm{K}$-and $\mu$-parameter value fluctuates within the certain ranges, according to the Table-1, [10-15]. The parameters of the AVR systems components [12-15] shall be specified in study $\mathrm{Ka}=10, \ddot{\mathrm{a}}=0.1, \mathrm{Ke}=1, \ddot{\mathrm{e}}$ $=0.4, \mathrm{Kg}=1$, òg $=1.0, \mathrm{Ks}=1$, and 's $=0.01$. This phase reaction to these unregulated AVR system parameters is seen in Figure 2. The results suggest that a big static error, a long settlement time and a high overflow have occurred. The AVR system must therefore improve the status and transient features of the region.

AVR System Stabilizer The application of a stabiliser is based mostly on greater relative stability in the
AVR system. For the stabiliser, $\mathrm{U}=[\mathrm{vR}], \mathrm{Y}=[\mathrm{VTI}]$, and $\mathrm{X}=[\mathrm{VT}$ VF VA VS VM], A, B, C and D matrices are indicated: Matrices:

$A=\left[\begin{array}{ccccc}-\frac{1}{\tau_{G}} & \frac{K_{G}}{\tau_{G}} & 0 & 0 & 0 \\ 0 & -\frac{1}{\tau_{E}} & \frac{K_{E}}{\tau_{E}} & 0 & 0 \\ 0 & 0 & -\frac{1}{\tau_{A}} & -\frac{K_{A}}{\tau_{A}} & \frac{K_{A}}{\tau_{A}} \\ 0 & \frac{K_{S}}{\tau_{S} \tau_{E}} & \frac{K_{E} K_{S}}{\tau_{S} \tau_{E}} & -\frac{1}{\tau_{S}} & 0 \\ \frac{K_{S}}{\tau_{S}} & 0 & 0 & 0 & -\frac{1}{\tau_{C}}\end{array}\right]$

(1)

$B=\left[\begin{array}{lllll}0 & 0 & \frac{K_{A}}{\tau_{A}} & 0 & 0\end{array}\right]^{T}$

$C=\left[\begin{array}{lllll}1 & 0 & 0 & 0 & 0\end{array}\right]^{T}$

The feature of the equation is:

$\Delta_{s}(S)=S^{5}+C_{4} S^{4}+C_{3} S^{3}+C_{2} S^{2}+C_{1} S+C_{0}$

where:

$C_{4}=\frac{1}{\tau_{A}}+\frac{1}{\tau_{S}}+\frac{1}{\tau_{E}}+\frac{1}{\tau_{C}}+\frac{1}{\tau_{C}}$

$C_{3}=\left(\frac{1}{\tau_{C}}+\frac{1}{\tau_{C}}\right)\left(\frac{1}{\tau_{A}}+\frac{1}{\tau_{S}}+\frac{1}{\tau_{E}}\right)+\frac{1}{\tau_{C} \tau_{C}}+\frac{1}{\tau_{S} \tau_{A}}+$ $\frac{1}{\tau_{E} \tau_{S}}+\frac{1}{\tau_{A} \tau_{E}}+\frac{K_{E} K_{S} K_{A}}{\tau_{A} \tau_{S} \tau_{E}}$

$C_{2}=\left(\frac{1}{\tau_{C}}+\frac{1}{\tau_{C}}\right)\left(\frac{1}{\tau_{A}}+\frac{1}{\tau_{S}}+\frac{1}{\tau_{E}}\right)+\frac{1}{\tau_{C} \tau_{C}}\left(\frac{1}{\tau_{A}}+\frac{1}{\tau_{S}}\right)+$ $\frac{K_{E} K_{S} K_{A}}{\tau_{A} \tau_{S} \tau_{E}}\left(\frac{1}{\tau_{C}}+\frac{1}{\tau_{E}}\right)+\frac{1}{\tau_{A} \tau_{S} T_{E}}$

$C_{1}=\frac{1}{\tau_{A} \tau_{S} \tau_{E}}\left(\frac{1}{\tau_{C}}+\frac{1}{\tau_{C}}\right)+\frac{K_{E} K_{S} K_{A}}{\tau_{A} \tau_{S} \tau_{E}}+\frac{1}{\tau_{C} \tau_{C}}\left(\frac{1}{\tau_{A} \tau_{S}}+\right.$ $\left.\frac{1}{\tau_{A} \tau_{E}}+\frac{1}{\tau_{S} \tau_{E}}\right)+\frac{R_{E} R_{S} R_{A}}{\tau_{C} \tau_{Q} \tau_{i} \tau_{S} T_{E}}$

$C_{1}=\frac{1+K_{C} K_{E} K_{C} K_{A}}{\tau_{C} \tau_{C} \tau_{A} \tau_{S} \tau_{E}}$

Transfer function of a Closed loop AVR system with stabiliser:

\section{Design of the Proposed FOPID Controller}

This section shows design process of a proposed major controller, i.e. FOPID controller AVR system. The model diagram of the controllers and their transfer-functions are given in Figure 3 which also includes traditional PID controllers. Based on model diagrams, conclusion is that there are three separate parameters for a standard PID controller, however the FOPID controller has different parameters. For each controller, the parameters have to be defined. 


$$
f(\theta)=\left(1-e^{\beta}\right)\left(M_{p}+E_{S S}\right)+e^{\beta}\left(t_{s}-t_{r}\right)(10)
$$

While the value of the value of $\beta=1$ and $M p$, ts, tr, is the over-shoot, set time, increase time and steady-state error percentages. os holds the parameters specified for the controller in Table 2 .

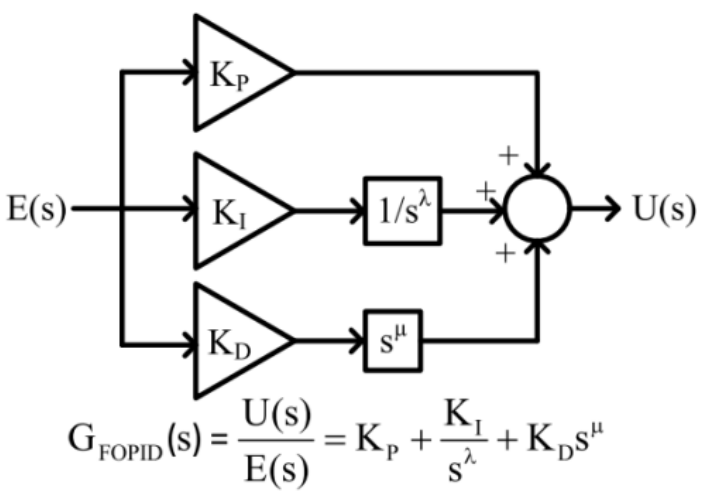

Fig. 1.. Block Diagram of FOPID

PID and FO PID are 10 times since an algorithm begins in a random initial position corresponding to the solutions for the design of the different controllers mentioned above. In general, a termination condition is established to end algorithm for an optimization process. For a variable, this can be a desired number value. When algorithm hits lower value in desired condition final condition can be considered fulfilled. However, such a circumstance is not optimal for optimising the control technology. Because even if the loop takes a very long duration, the algorithm cannot achieve the desired value. Tens of thousands and hundreds. The algorithm can therefore be employed several times $(1,5,10,15 \ldots$...and more) as a further method of system optimization and the optimum system iteration is provided. The powerful metaheuristic algorithms can handle random original position issue in a short search area satisfactorily. For each suggested controller ten separate studies are included in this analysis. In these 10 tests, the value of the cost function is so close that the control parameter value is optimally obtained. Should there not be a substantial difference between these 10 trials. The Table 3 additionally provides optimised controller settings, covering and comparing various PID and FOPID controllers of the research.

\section{The Change of the Stabilizer Parameters}

The control loop has 3 times TE, TA and TG constant with a rate feedback. A big advantage of the Rate Feedback System is that the controller adds zero to AVR Open loop of the transfer function, which can improve relative stability. The stabiliser loop is a feedback which boosts the circuit's reactivity and stability. In the AVR stabiliser system the continuous response is equated as:

$V_{T}(\infty)=\frac{K_{E} K_{C} K_{A}}{1+K_{C} K_{E} K_{C} K_{A}}$

Roots of feature AVR system equation with stabilisers are provided in table $\mathrm{V}$ with three alternative values, $\mathrm{Ks}$ and Ts. Simulation results for Ks and Ts changes are shown in Figs. 9 and 10. The results show that the variation $\mathrm{Ks}$ and TS impacts the curve maximum, adjustment times, or overflow, but that it does not affect the final terminal voltage values of the stable and unstable systems. A higher K's value and a lower TS result in quick exceedance and settlement time. Increasing the time of the stabiliser decreases the time of both increase and peak. In contrast, increasing stabiliser loop gain increases both higher and higher times and reduces higher gain percentages.

\section{Fractional Order PID Controller}

A Calculus Fractional FOPID controller provides non integer command differentiation and integration. These non-triggers give greater flexibility for design in different fields of engineering including theory of control, signal processing, robotics, etc. This attracted the attention of scholars, especially in the control system sector, to the fractional computation. Riemann-Livouville, Grunwald-Letnikov and Caputo are famous and widely used for their definitions. In addition, approximation approaches are frequently favoured because it is difficult to calculate the definitions presented. One of these approximative approaches is the Oustaloup approximation method for converging fractional order transfer functions.

This work uses the Oustaloup procedure for the approximation of the transfer function to produce fractional transmission functions in the frequency field $\mathrm{w}$ directories [10-5 105] r/s.

In the form of a PID $\mu$ is written a FOPID controller containing the fractional integrator, with $c$ and $\mu$ as the integrator's and the differentiator's commands. (1) represents a general FOPID controller transfer function with $\mathrm{KP}, \mathrm{KI}$ and $\mathrm{KD}$ proportional, integral and derivative gains, respectively, whose block diagram is illustrated in Fig. $3 \mathrm{~d}$.

$G_{F O P I D}(s)=K_{p}+\frac{K_{l}}{s^{\lambda}}+K_{D} S^{\mu}$

where $\beta$ and $\mu$ are, respectively, the order of the integrator and differentiator. The FOPID controller contains two additional settings, i.e. $\mu$ and $\beta$, to be tuned, different from the typical PID controller. While, these external parameters enable more control system design possibilities, they improve 
complexity of controller and make tweaking of parameters highly complex than normal PID controllers.

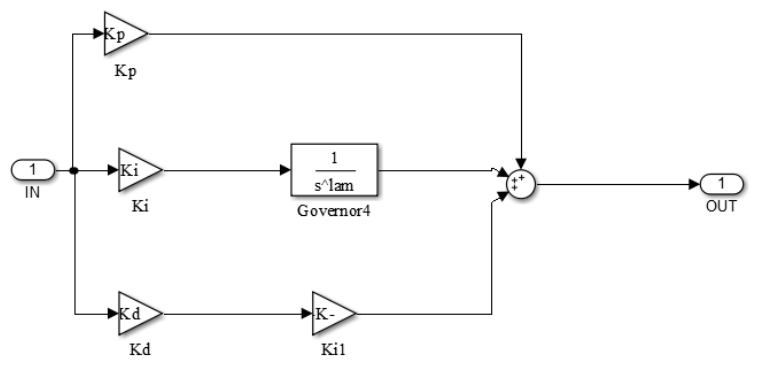

Fig. 2. Simulation of FOPID

Table 1 AVR system Transfer functions components

\begin{tabular}{lllll}
\hline & Transfer function & \multicolumn{2}{l}{ Limits of parameters } & Used parameters \\
\cline { 3 - 5 } & & Gain & Time constant $(s)$ & \\
\hline Amplifier & $T(s)=K_{a} /\left(1+\tau_{a} s\right)$ & $10 \leqslant K_{a} \leqslant 40$ & $0.02 \leqslant \tau_{a} \leqslant 0.1$ & $K_{a}=10, \tau_{a}=0.1$ \\
Exciter & $T(s)=\frac{K_{e}}{1+\tau_{e} s}$ & $1 \leqslant K_{e} \leqslant 10$ & $0.4 \leqslant \tau_{e} \leqslant 1.0$ & $K_{e}=1, \tau_{e}=0.4$ \\
Generator & $T(s)=\frac{K_{g}}{1+\tau_{g} s}$ & $0.7 \leqslant K_{g} \leqslant 1.0$ & $1.0 \leqslant \tau_{g} \leqslant 2.0$ & $K_{g}=1, \tau_{g}=1.0$ \\
Sensor & $T(s)=\frac{K_{s}}{1+\tau_{s} s}$ & $0.9 \leqslant K_{s} \leqslant 1.1$ & $0.001 \leqslant \tau_{s} \leqslant 0.06$ & $K_{s}=1, \tau_{s}=0.01$ \\
\hline
\end{tabular}

\section{Results}

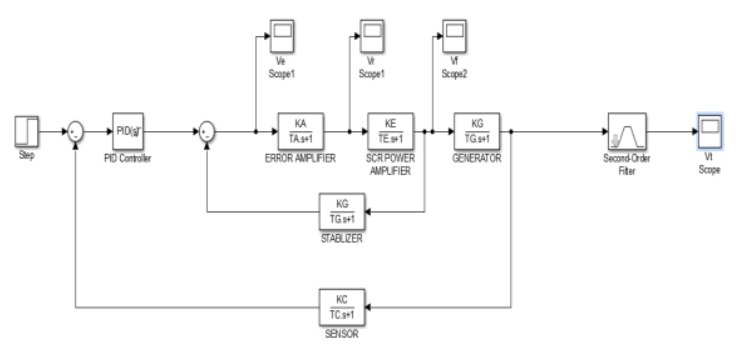

(a)

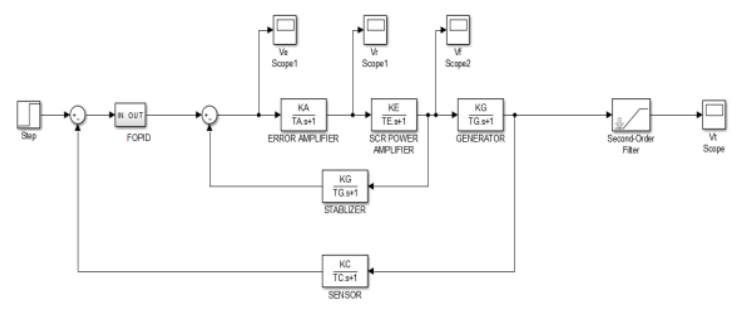

(b)

Fig3. Transfer function simulations of the controllers. a: PID, b:FOPID

$\mathrm{TG}=4$;

$\mathrm{KG}=0.7$;

$\mathrm{KA}=5$;

$\mathrm{TA}=0.02$;

$\mathrm{KE}=10$;

$\mathrm{TE}=0.5$;

$\mathrm{KS}=0.02$;

$\mathrm{TS}=0.013$

$\mathrm{TC}=0.001$;
$\mathrm{KC}=0.1$;

$\mathrm{Kp}=0.1$;

$\mathrm{Ki}=0.02$;

$\mathrm{Kd}=0.05$;

$\mathrm{s}=1$;

$\operatorname{lam}=3$;

$\mathrm{u}=0.1$;

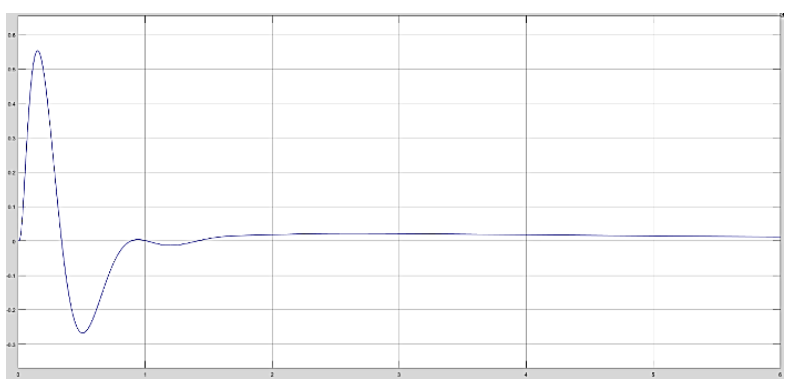

Fig. 4. AVR system terminal voltage step response with PID, stabilizer (solid) and without compensation (dash) with 0.5 amplitude with time period $1 \mathrm{sec}$ and from 2 secs amplitude doesn't settle down to zero.

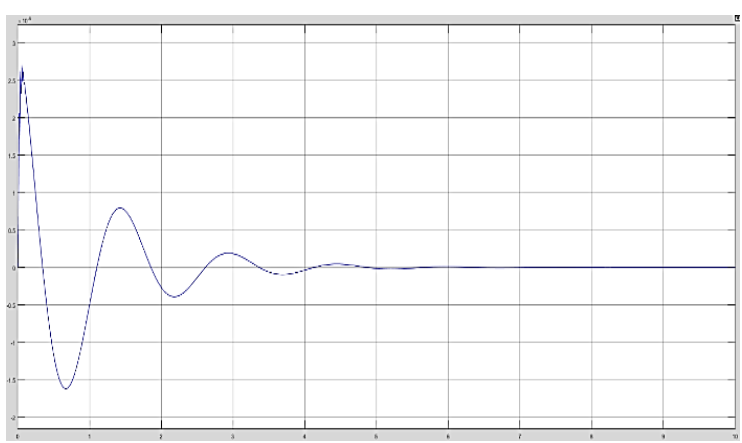


Fig. 5 AVR system terminal voltage step response with FOPID , stabilizer (solid) and without compensation (dash) with amplitude of $0.2 \times 10^{-5}$ in 5 secs.

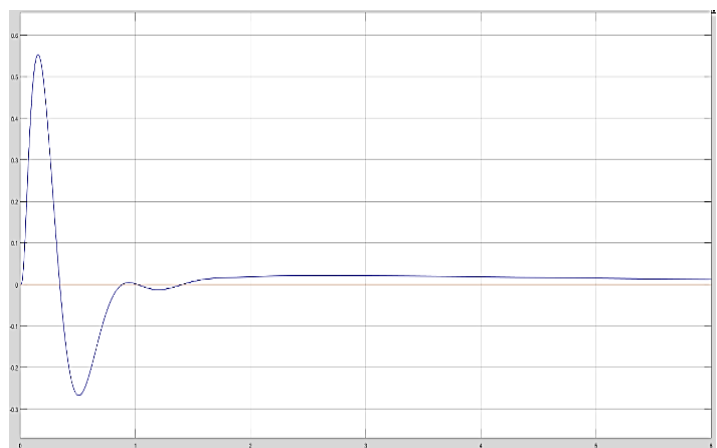

Fig. 6 AVR system terminal voltage step response with PID and FOPID, stabilizer (solid) and without compensation (dash)

\section{Conclusion}

The objective of the present research is to develop regulatory performance of the normally used AVR system with the single fractional filter FOPID controller. This study also seeks to prove the efficiency of proposed FOPID controller and to make the suggested control of fractional filter a beneficial impact. For the identical AVR system the PID and FOPID controllers, which perfectly tuned their literature settings, are applied. Time domain analysis are performed for various controllers and superiority of suggested controller is exposed. Box diagrams analyse AVR system stability with different controllers. In addition, the durability of their designed controllers is separately examined by both the intravenous parameters of AVR system and external disorder injecting into AVR system. Consequently, results of research analyses that their fractional filter by proposed FOPID controller improved substantively regulatory performance of AVR system. Finally, AVR system was simulated and several parameter effects are examined.

\section{References}

1. Ortiz-Quisbert ME, Duarte-Mermoud MA, Milla F, Castro-Linares R, Lefranc G. (2018).

2. Gaing Z-L. IEEE Trans Energy Convers (2004).

3. Bingul Z, Karahan O. J Franklin Inst B (2018).

4. Tang Y, Cui M, Hua C, Li L, Yang Y. Expert SystAppl (2012)

5. Zeng G-Q, Chen J, Dai Y-X, Li L-M, Zheng C-W, Chen (2015).

6. Gupta T, Sambariya . IEEE; 1 (2017).
7. Ribeiro R, Neto C, Costa F, Rocha T, Barreto $\mathrm{R}$ (2015).

8. Ayas MS.. ElectrEng (2019).

9. Ekinci S, Hekimoğlu B. IEEE Access (2019).

10. Gozde H, Taplamacioglu M. Comparative J Franklin Inst B (2011)

11. Sahib MA, Ahmed BS. J Adv Res (2016).

12. Bendjeghaba O.. J ElectrEng (2014).

13. Çelik E, Durgut R, Eng Sci Technol Int J (2018).

14. Ekinci S, Hekimoğlu B, Kaya S. ICAIDP. IEEE; (2018).

15. Anbarasi S, Muralidharan S. Enhancing the (2016).

16. Duman S, Yörükeren N, Altaş İH. Turk J ElectrEngComput Sci, Elektr (2016)

17. Pradhan R, Majhi SK, Pati BB. World J Eng (2018).

18. Mohanty PK, Sahu BK, Panda S.. Electr Power ComponSyst (2014).

19. Rani, V.U., Sridevi, J., Sai, P.M., SeFet (2021)

20. Sridevi, J., Amarnath, J., Govinda Rao, G., International J of App. Engg Research, (2012)

21. Rajagiri, A.K., Mn, S.R., Nawaz, S.S., Suresh Kumar, T, E3S Web of Conferences, 84 (2019)

22. B. J. Varghese, P. B. Bobba and M. Kavitha, 2016 IEEE 7th Power India International Conference (PIICON), 2016, pp. 1-6

23. B. J. Varghese and P. B. Bobba, " 2016 IEEE 1st International Conference on Power Electronics, Intelligent Control and Energy Systems (ICPEICES), 2016, pp. 1-5 